\title{
Retos de la implementación de RDA en las bibliotecas de América Latina
}

\author{
Filiberto Felipe Martínez Arellano* \\ * Universidad Nacional Autónoma de México. Instituto de Investigaciones Bibliotecológicas y \\ de la Información, México | felipe@unam.mx
}

El inicio de los trabajos para el desarrollo del nuevo estándar de catalogación RDA (Resource Description and Accces) se remontan al año de 2004; sin embargo, como parte del Plan Estratégico 2007-2009 de la Sección de Catalogación de la Federación Internacional de Asociaciones e Instituciones Bibliotecarias (IFLA por sus siglas en inglés), se efectuaron paralelamente una serie de reuniones denominadas IFLA Meetings of Experts on an International Cataloguing Code, para cumplir con uno de los objetivos de dicho plan estratégico: "promover el desarrollo de un código internacional de catalogación para el acceso y la descripción bibliográfica" (IFLA. Cataloging Section, 2016). Estas reuniones tuvieron lugar en Frankfurt, Alemania (2003), Buenos Aires, Argentina (2004), el Cairo, Egipto (2005), Seúl, Corea (2006) y Pretoria, Sudáfrica (2007).

La segunda de esta serie de reuniones, celebrada el 17 y 18 de agosto en Buenos Aires Argentina, tuvo como objetivo: "Revisar y actualizar el borrador de la Declaración de Principios [de Catalogación] elaborado en 2003 en la reunión europea, así como discutir los códigos de catalogación actualmente en uso en América Latina para comparar sus similitudes y diferencias, a fin de lograr acercamientos para el posible desarrollo de un Código de Catalogación Internacional". A esta reunión asistieron 76 catalogadores provenientes de los siguientes países de América Latina y el Caribe: Anguila, Antigua \& Barbuda, Argentina, Aruba, Bahamas, Barbados, Belice, Bolivia, Brasil, Chile, Colombia, Costa Rica, Cuba, Curazao, Ecuador, El Salvador, Guatemala, Guyana, Haití, Honduras, Jamaica, México, Nicaragua, Panamá, Perú, Puerto Rico, República Dominicana, St. Maarten, Surinam, Trinidad \& Tobago, Uruguay y Venezuela (IFLA. Cataloging Section, 2016a)

Esta reunión fue uno de los elementos clave que despertaron el interés entre los catalogadores de América Latina y el Caribe por conocer las características del nuevo estándar de catalogación, así como discutir la problemática de su implementación en las bibliotecas de la región. Después de la reunión en Buenos Aires, tuvieron lugar la celebración de los Encuentros Internacionales de Catalogadores en diferentes países de la región, los cuales han contado con 
la participación de especialistas del continente, además de otros con un reconocido prestigio internacional, destacándose la presencia de Barbara Tillet y Ana Cristán de la Biblioteca del Congreso de los Estados Unidos. Estos encuentros han sido celebrados en los siguientes países:

\author{
I Lima, Perú (2005) \\ II Ciudad de México, México (2006) \\ III Buenos Aires, Argentina (2007) \\ IV Bogotá, Colombia (2008) \\ V Santo Domingo, República Dominicana (2009) \\ VI San José, Costa Rica (2010) \\ VII Buenos Aires, Argentina (2011) \\ VIII Caracas, Venezuela (2012) \\ IX São Paulo, Brasil (2013) \\ X La Paz, Bolivia (2015)
}

Indudablemente, las memorias de esta serie de encuentros proporcionan diferentes perspectivas de los retos que implica la adopción e implementación de RDA en las bibliotecas de los países de la región.

Adicionalmente, la segunda reunión de la serie IFLA Meetings of Experts on an International Cataloguing Code, motivó el surgimiento de encuentros nacionales de catalogación, los cuales fueron celebrados primeramente en México (ocho ocasiones 2004-2014) y en Argentina (cinco ocasiones, 2008-2015), y posteriormente en Colombia (dos ocasiones, 2009-2010), Brasil (tres ocasiones, 2012-2014) y Venezuela (tres ocasiones, 2013-2016). En estos eventos, los especialistas de cada uno de los respectivos países han abordado la problemática de la adopción e implementación de RDA, presentando documentos de gran importancia, los cuales han sido publicados en las memorias correspondientes, muchas de ellas disponibles en la red.

Por otro lado, es importante hacer mención a los grupos de trabajo sobre RDA que se han formado en algunos países, siendo los siguientes: Grupo de trabajo sobre RDA (Argentina); Grupo de Interés RDA (Chile), GIRCH; Foro RDA (Biblioteca Nacional de Colombia); y Seminario de Organización de la Información del Instituto de Investigaciones Bibliotecológicas de la UNAM, en donde además de este instituto participan la Biblioteca Nacional de México y la Dirección General de Bibliotecas de la UNAM.

Con base en lo expuesto, es evidente que en América Latina ha existido un gran interés entre los catalogadores por conocer el nuevo estándar de catalogación RDA, así como por analizar los retos que implica su implementación en las bibliotecas de la región. En este sentido, se consideró conveniente elaborar este dossier, el cual ofrece un panorama sobre los retos que ha implicado la implementación de las RDA en tres instituciones de América Latina. La experiencia 
de estas tres instituciones sobre la implementación de RDA puede ser utilidad para otras bibliotecas de la región. Adicionalmente, es conveniente mencionar que los documentos generados en los grupos de trabajo de estas instituciones, muchos de los cuales se encuentran en acceso abierto, también podrán ser de utilidad para aquellas bibliotecas latinoamericanas que se encuentran en el proceso del conocimiento y aplicación de RDA.

\section{Referencias bibliográficas}

IFLA. Cataloging Section (2016). IFLA Meetings of Experts on an International Cataloguing Code. Recuperado de http://www.ifla.org/node/576

IFLA. Cataloging Section (2016a). Second IFLA Meeting of Experts on an International Cataloguing Code. Recuperado de http://www.ifla.org/node/10599 